\title{
Comparison of different sufentanil-tramadol combinations for pain relief within the first 24 hours after cesarean section:
} a retrospective study

This article was published in the following Dove Press journal:

Journal of Pain Research

\section{Xueqin Cao \\ Xianwei Zhang}

Department of Anesthesiology, Tongji Hospital of Tongji Medical College, Huazhong University of Science and Technology, Wuhan, People's Republic of China
Correspondence: Xianwei Zhang Department of Anesthesiology, Tongji Hospital of Tongji Medical College, Huazhong University of Science and Technology, Wuhan, People's Republic of China

Tel +86 2783663173

$\mathrm{Fax}+862783662853$

Email painfree@qq.com
Introduction: Postcesarean section pain management is important for both the mother and the newborn. This study compared the analgesic effects and incidence of adverse events associated with intravenous patient-controlled analgesia (iv-PCA), using different sufentanil-tramadol combinations for postoperative pain control.

Methods: Parturients $(n=5,794)$ who had been scheduled for cesarean section under neuraxial anesthesia and had received iv-PCA between September 2013 and March 2017 were retrospectively analyzed. These patients were assigned to three groups, based on different sufentanil-tramadol combinations: ST1 $(n=1,347)$, ST2 $(n=2,401)$, and ST3 $(n=2,046)$. The analgesic efficacy, total drug consumption, and incidence of adverse effects within 24 hours after surgery were compared among the three groups.

Results: The ST3 group had lower visual analog scale pain scores at rest and with movement at all time points during the first 24 hours postoperatively than the other two groups $(P<0.01$, Bonferroni corrected). The sufentanil dosage administered to the ST3 group was lower, and the tramadol dosage was higher than those administered to the other groups within 24 hours after surgery $(P<0.01$, Bonferroni corrected). Moreover, all parturients scored 2 points on the Ramsay sedation scale. Adverse reactions such as pruritus and respiratory depression were not observed in any group. No significant differences were noted in the incidence of nausea/vomiting, abdominal distension, and dizziness among the three groups $(P>0.05)$.

Conclusion: The visual analog scale scores for postoperative pain decreased as the concentrations of sufentanil and tramadol administered in iv-PCA moderately increased over 24 hours after surgery. This analgesic strategy resulted in a significant reduction in the total sufentanil requirement without increasing the incidence of adverse effects.

Keywords: patient-controlled analgesia, sufentanil, tramadol, VAS score, cesarean section

\section{Introduction}

Cesarean sections (CSs) are one of the most common surgical procedures performed in female patients. A recent study revealed that women post-CS ranked ninth for pain severity among 179 different surgical procedures. ${ }^{1}$ Uncontrolled pain may interfere both with the mother's ability to care optimally for her child and the early stage of mother-child communication. ${ }^{2}$ Therefore, appropriate analgesia is important in the short term, following a CS.

The most common methods of pain management after CS include neuraxial medications (intrathecal opioids, and continuous and patient-controlled epidural 
infusions) and intravenous analgesics (acetaminophen, nonsteroidal antiinflammatory drugs [NSAIDs], and intravenous opioids). ${ }^{3}$ However, there is presently no "gold standard" for postcesarean birth analgesia. ${ }^{4}$ Each approach has its limitation. Although intrathecal morphine is thought to be the best single-shot drug for postcesarean pain, it is associated with numerous side effects, especially pruritus and vomiting. ${ }^{5,6}$ Moreover, the use of continuous and patient-controlled epidural analgesia (PCEA) could decrease maternal mobility, complicate anticoagulation prophylaxis, and increase nursing workload. ${ }^{7}$ Since the use of intravenous patient-controlled analgesia (iv-PCA) in obstetrics was first described in 1976, it has been widely used, as the patient-controlled aspect of this approach achieves greater patient satisfaction than other methods. ${ }^{8}$ Morphine, hydromorphine, fentanyl, and sufentanil are popular opioids frequently used in iv-PCA. ${ }^{9-11}$

Sufentanil, a selective $\mu$-receptor agonist, is ideal for ivPCA because of its fast onset and short duration of action, and strong analgesic effect, with a lower incidence of respiratory depression than morphine, alfentanil, and fentanyl. ${ }^{9,12}$ It has been in common use in the People's Republic of China for postoperative pain management. ${ }^{12,13}$ Because of the two components of pain following $\mathrm{CS}$, somatic pain from the wound itself and visceral pain arising from the uterus, sufentanil alone is insufficient to produce the desired relief in parturients, and higher doses could lead to severe adverse reactions such as respiratory depression. ${ }^{14}$ Tramadol, as a weak $\mu$-opioid receptor agonist, also inhibits presynaptic noradrenaline and serotonin $(5-\mathrm{HT})$ reuptake, stimulates 5-HT secretion, and shows good clinical efficacy in treating visceral pain without significant side effects. ${ }^{15-17}$ However, for severe postoperative pain, the use of tramadol as the sole analgesic is not recommended. ${ }^{6}$ Thus, the concomitant use of tramadol with other opioids may provide a synergistic effect that is beneficial and advisable in the treatment of severe postoperative pain and could reduce the need for other opioids. ${ }^{18}$

Sufentanil-tramadol combinations for iv-PCA have been used in post-CS pain management since September 2013 at our institution. Limited studies exist in the literature that compare the effects of different sufentanil-tramadol combinations as analgesic agents post-CS. Therefore, we retrospectively evaluated and compared postoperative analgesic efficacy, drug consumption, and adverse effects with different sufentanil-tramadol combinations in iv-PCA on patients who had undergone CSs.

\section{Methods}

This retrospective study was approved by the Research and Ethics Committee of Tongji Hospital at Tongji Medical
College, Huazhong University of Science and Technology. The requirement for written informed consent was waived for this medical record-review study under the regulations of the Institutional Review Board (IRB). Patient records and related information were anonymized before analysis. On receipt of IRB approval, data were compiled from a postoperative analgesic follow-up database that was maintained by the Acute Pain Service (APS) at our hospital ${ }^{13}$ between September 2013 and March 2017. Parturients between the ages of 18 and 50 years, with an American Society of Anesthesiologists physical status score of I to III, who had undergone a CS under neuraxial anesthesia were enrolled. The exclusion criteria for participation were as follows: received general anesthesia; received intraoperative sedation or additional opioids; the presence of severe hepatic or kidney disease; transferred to the intensive care unit postoperatively; and incomplete patient charts.

Our primary outcome was the quality of postoperative pain relief provided by different sufentanil-tramadol combinations. The secondary outcome was the manifestation of any side effects within 24 hours after cesarean delivery for different sufentanil-tramadol combinations.

Among 6,538 patients evaluated for eligibility, 5,794 patients were included in this study and assigned to three groups to which different sufentanil-tramadol combinations were administered as follows: $1 \mu \mathrm{g} / \mathrm{mL}$ sufentanil and 1.4 $\mathrm{mg} / \mathrm{mL}$ tramadol diluted to $150 \mathrm{~mL}$ with normal saline (ST1, $\mathrm{n}=1,347) ; 1 \mu \mathrm{g} / \mathrm{mL}$ sufentanil and $2 \mathrm{mg} / \mathrm{mL}$ tramadol diluted to150 mL with normal saline (ST2, $\mathrm{n}=2,401$ ); and $1.3 \mu \mathrm{g}$ / $\mathrm{mL}$ sufentanil and $4 \mathrm{mg} / \mathrm{mL}$ tramadol diluted to $150 \mathrm{~mL}$ with normal saline (ST3, n=2,046; Figure 1).

Neuraxial anesthesia for CS is standardized at our institution. Furthermore, combined spinal-epidural anesthesia is routinely used. Standard dosing included $1.6-1.8 \mathrm{~mL}$ of $0.5 \%$ hyperbaric bupivacaine. No intrathecal opioids were administered. The epidural catheter was placed as soon as the spinal needle was withdrawn. All cesarean deliveries were performed using a Pfannenstiel incision.

Immediately after surgery, iv-PCA was commenced through a PCA device. The PCA parameters were set according to the body weight of each patient. For example, patients weighing 40-50 kg received a background infusion at a rate of $1 \mathrm{~mL} /$ hour, a $1 \mathrm{~mL}$ bolus injection, and PCA with a $10 \mathrm{~min}$ utes lockout interval and limit of $10 \mathrm{~mL} /$ hour (Table 1). The iv-PCA was continued for 24 hours.

Postoperative analgesia was managed by the APS. Parturients were followed-up at 4-6 hours, 8-12 hours, and 18-24 hours postoperatively to evaluate the following parameters: vital signs, pulse oxygen saturation $\left(\mathrm{SpO}_{2}\right)$, respiratory rate, 


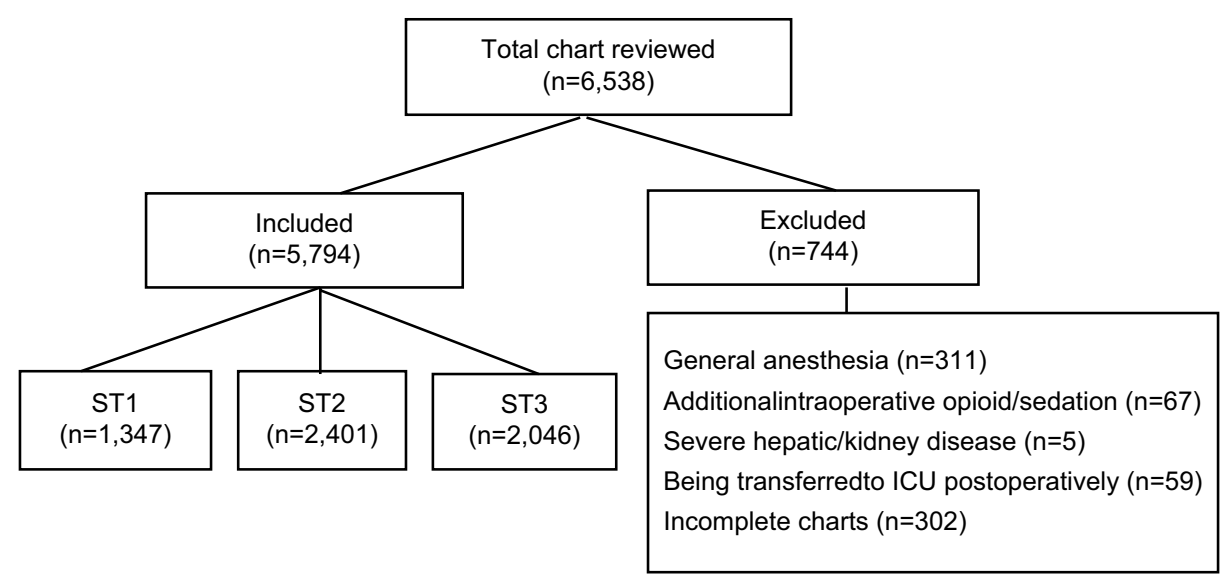

Figure I Inclusion and exclusion flowchart.

Table I Patient-controlled analgesia protocol

\begin{tabular}{lllll}
\hline Weight (kg) & $\begin{array}{l}\text { Background infusion } \\
(\mathbf{m L} / \text { hour) }\end{array}$ & $\begin{array}{l}\text { Bolus injection } \\
(\mathbf{m L})\end{array}$ & $\begin{array}{l}\text { Lockout interval } \\
\text { (minutes) }\end{array}$ & $\begin{array}{l}\text { Limit } \\
(\mathbf{m L} / \mathrm{hour})\end{array}$ \\
\hline $40-50$ & 1 & 1 & 10 & 10 \\
$50-60$ & 1.5 & 1.5 & 10 & 12 \\
$60-70$ & 2 & 1.5 & 10 & 15 \\
$>70$ & 2 & 2 & 10 & 15 \\
\hline
\end{tabular}

pain score, sedation score, bolus attempts, sufentanil or tramadol consumption, and adverse effects such as postoperative nausea and vomiting (PONV), respiratory depression, abdominal distention, pruritus, urinary retention, and dizziness. Maternal age, height, weight, and PCA parameters were also recorded. Pain was assessed using a $100-\mathrm{mm}$ visual analog scale (VAS; from 0 , no pain to 100 , the worst imaginable pain) both during movement and at rest. A VAS score $\geq 40$ was used to identify patients with inadequate analgesia. ${ }^{19}$ Sedation was assessed using the Ramsay sedation scale (RSS; 1 , anxious patient; 2 , cooperative and tranquil; 3 , responding to command; 4, brisk response to stimulus; 5, sluggish response to stimulus; and 6 , no response to stimulus). ${ }^{12} \mathrm{~A}$ verbal descriptive scale was used to define PONV (where $0=$ none, $1=$ mild, $2=$ moderate, and $3=$ severe nausea) ${ }^{20}$ Respiratory depression was defined as a respiratory rate $<10 /$ minute and $\mathrm{SpO}_{2}<90 \%{ }^{21}$ As all parturients who have undergone a CS also have an indwelling catheter until 24 hours postoperatively at our institution, urinary retention was not recorded.

If the VAS $<40$, the parturient continued to receive the same analgesic protocol; if the VAS $\geq 40$, PCA parameters were upregulated by members of the APS, and pain was assessed again after 10 minutes. If the parturient continued to complain about inadequate analgesia, flurbiprofen axetil was administered. If parturients complained of severe adverse events such as respiratory depression or PONV, the PCA parameters were adjusted accordingly by the APS.

\section{Statistics}

Descriptive statistics were computed for all study variables. The Kolmogorov-Smirnov test and normal quantize plots were used to determine whether continuous variables were normally distributed. Normally distributed data were analyzed using one-way analysis of variance. Nonnormally distributed data were analyzed using the Kruskal-Wallis test. Dichotomous variables were expressed in proportions and analyzed using the chi-squared test. A $P$-value $<0.05$ was considered significant. Comparisons between two groups were adjusted using the Bonferroni correction. An adjusted $P$-value $<0.01$ was considered significant. Numerical calculations were performed using the SPSS software (Version 16.0, SPSS Inc., Chicago, IL, USA). 


\section{Results}

The pain scores of study participants are presented in Figure 2. In comparison with the other groups, the ST3 group had lower VAS pain scores, both at rest and with movement, at all time points during the first 24 hours postoperatively. In addition, the VAS pain score in the ST2 group was lower than that in the ST1 group at rest $(P<0.01$, Bonferroni corrected). With movement, the VAS scores in the ST2 group were higher than those in the ST1 group at 8-12 hours postoperatively; however, no significant differences were noted at 4-6 hours and 18-24 hours after surgery $(P<0.01$, Bonferroni corrected). Table 2 shows the number of bolus attempts and consumption of sufentanil and tramadol during the first 24 hours after surgery. Generally, the bolus attempts and sufentanil consumption in the ST3 group were lower than those in the other groups. However, a higher number of bolus attempts were made in the ST3 group than in the
ST2 group at 4-6 hours postoperatively $(P<0.01$, Bonferroni corrected). Moreover, the quantity of tramadol administered in the three groups increased with decreasing sufentanil consumption $(P<0.01$, Bonferroni corrected). The RSS score of all parturients was 2 points.

The incidence of inadequate analgesia among the three groups differed significantly. At rest, the ST3 group showed lower levels of inadequate analgesia at 8-12 hours and 18-24 hours postoperatively, in comparison with the other study groups $(P<0.01$, Bonferroni corrected; Figure 3$)$. With movement, the incidence of inadequate analgesia in the ST3 group was lower than that in the other groups at 4-6 hours and 18-24 hours postoperatively $(P<0.01$, Bonferroni corrected; Figure 4).

During follow-up, adverse reactions, such as pruritus and respiratory depression, were not observed in any of the three groups. No significant differences were noted in the
A

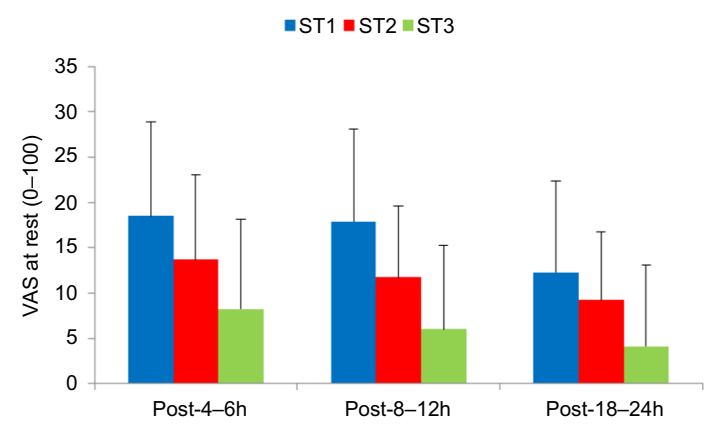

B

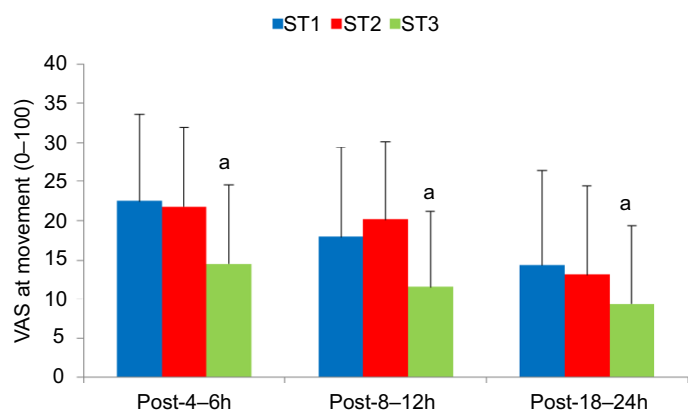

Figure 2 Pain score during the first 24 hours after surgery.

Notes: Data are expressed as mean \pm SD. (A) Pain score at rest. $P<0.01$ among the three groups; (B) Pain score with movement. ${ }^{a}<<0.01$ vs other groups. No significant differences were noted between STI and ST2 at 4-6 hours and 18-24 hours postoperatively; $P<0.01$ between STI and ST2 at 8-12 hours postoperatively.

Table 2 Bolus attempts and consumption of sufentanil and tramadol

\begin{tabular}{|c|c|c|c|c|}
\hline Variables & $\begin{array}{l}\text { STI } \\
(n=1,347)\end{array}$ & $\begin{array}{l}\text { ST2 } \\
(n=2,40 I)\end{array}$ & $\begin{array}{l}\text { ST3 } \\
(n=2,046)\end{array}$ & $P$-value \\
\hline \multicolumn{5}{|l|}{ Bolus attempts (n) } \\
\hline 4-6 hours postoperatively & $2(3)$ & I (3) & $2(3)$ & $<0.05$ \\
\hline $8-12$ hours postoperatively & $6(9)$ & $5(9)$ & $4(6)$ & $<0.05$ \\
\hline 18-24 hours postoperatively & $8(8)$ & $7(11)$ & $6(9)$ & $<0.05$ \\
\hline \multicolumn{5}{|l|}{ Sufentanil consumption $(\mu \mathrm{g})$} \\
\hline 4-6 hours postoperatively & $21(15)$ & $21(15)$ & $20(14)$ & $<0.05$ \\
\hline $8-12$ hours postoperatively & $69(3 \mathrm{I})$ & $65(28)$ & $55(24)$ & $<0.05$ \\
\hline I8-24 hours postoperatively & $95(36)$ & $97(37)$ & $83.2(30)$ & $<0.05$ \\
\hline \multicolumn{5}{|l|}{ Tramadol consumption (mg) } \\
\hline 4-6 hours postoperatively & $29.4(21)$ & $42(30)$ & $60(44)$ & $<0.05$ \\
\hline $8-12$ hours postoperatively & $96.6(43.4)$ & $130(56)$ & $168(72)$ & $<0.05$ \\
\hline 18-24 hours postoperatively & $133(50)$ & $194(74)$ & $256(92)$ & $<0.05$ \\
\hline
\end{tabular}

Notes: Data are expressed as $M\left(Q_{R}\right)$. Data were compared using the Kruskal-Wallis test among three groups. ${ }^{\text {a }}$-value $<0.05$ was considered significant. Data were compared using the Bonferroni correction between two groups. An adjusted $P$-value $<0.0$ I was considered significant. 
incidence of nausea/vomiting, abdominal distension, or dizziness among the three groups ( $P>0.05$; Figure 5$)$.

\section{Discussion}

The present study showed that the protocol used in the ST3 group controlled pain more effectively than those used in the other groups. Although the VAS scores for pain with movement in the ST2 group were higher than those in the ST1 group at 8-12 hours postoperatively, no significant differences were noted at 4-6 hours and 18-24 hours after surgery. The VAS scores for postoperative pain declined as the concentrations of sufentanil and tramadol were moderately increased within 24 hours after surgery. Moreover, the total consumption of sufentanil was reduced, without any increase in the incidence of side effects. Thus, postoperative analgesic effects could be improved by adjusting the concentrations of

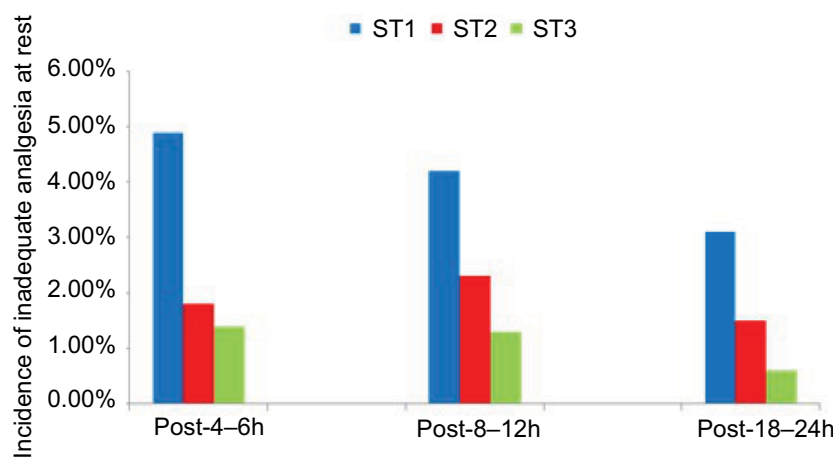

Figure 3 Incidence of inadequate analgesia among the three groups at rest after surgery.

Notes: At Post-4-6h, ST2 or ST3 vs STI $(P<0.01)$. No significant differences were noted between ST2 and ST3; $P<0.01$ among the three groups at $8-12$ hours and 18-24 hours postoperatively. sufentanil-tramadol combinations. However, a high dose of tramadol is associated with serious adverse events, particularly seizures and nausea/vomiting. ${ }^{22}$ The dose of tramadol used in our study was within the safe range. ${ }^{23}$ Our study also showed no significant differences in the incidence of nausea/ vomiting among the three groups. These results are consistent with another study conducted at our institution, which reported no significant differences in nausea/vomiting scores between a sufentanil iv-PCA group and a tramadol iv-PCA group. ${ }^{20}$ The incidence of side effects in the present study was also similar to that reported in the study of Demirel et al. ${ }^{24}$

The U.S. Food and Drug Administration issued a safety announcement for tramadol in April 2017 that cautioned against the use of tramadol in nursing mothers and the potential risk of serious adverse reactions, including respiratory depression and even death. ${ }^{25}$ However, although tramadol

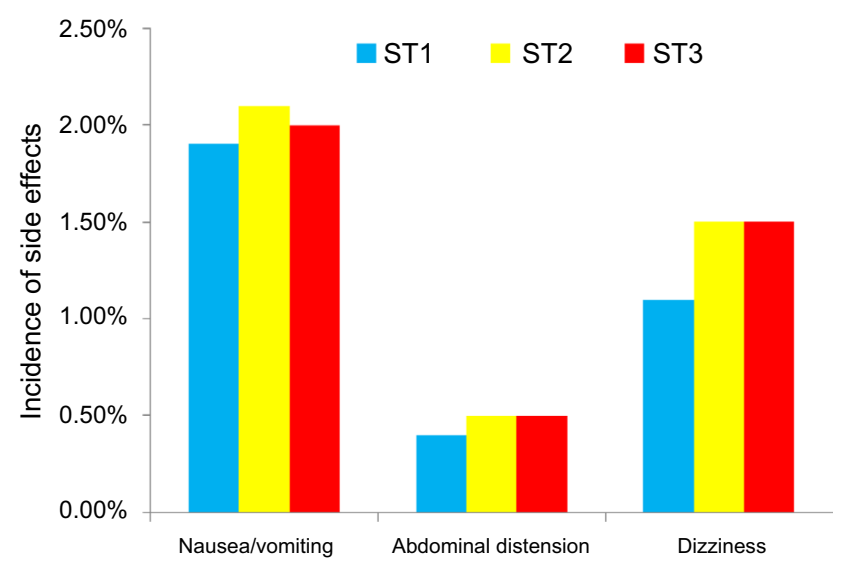

Figure $\mathbf{5}$ Incidence of side effects among three groups after surgery. Note: No significant differences were noted among the three groups $(P>0.05)$.

$\approx \mathrm{ST} 1=\mathrm{ST} 2=\mathrm{ST} 3$

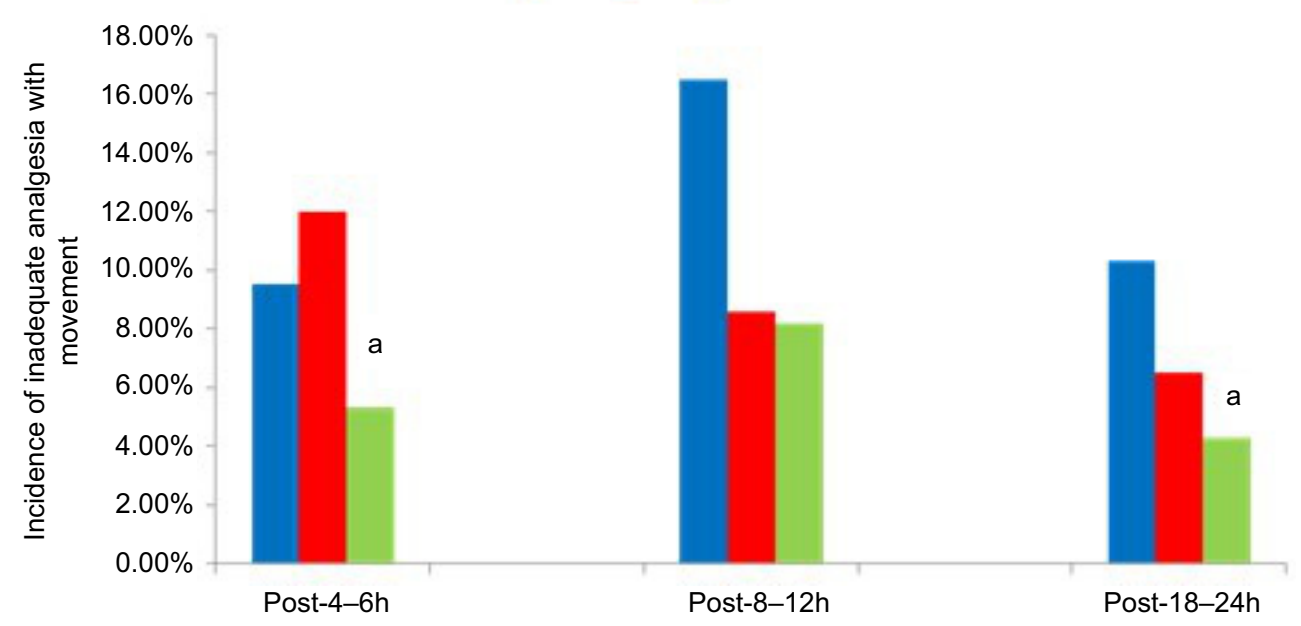

Figure 4 Incidence of inadequate analgesia while changing positions among the three groups after surgery.

Notes: ${ }^{P}<0.01$ vs other groups; $P<0.0$ I between STI and ST2 at any time point. No significant differences were noted between $S T 2$ and $S T 3$ at $8-12$ hours postoperatively. 
is a synthetic analog of codeine, the two are not quite the same. Tramadol exerts its analgesic activity via at least two complementary mechanisms. It has a lower risk of respiratory depression compared with classical opioids, and respiratory depression does not normally occur when the drug is used within the therapeutic range. The Change Pain Latin America panel agreed that tramadol is well tolerated, and without the safety issues associated with long-term NSAIDs use, has fewer opioid-like side effects than classical opioids, and a lower risk of abuse. ${ }^{26}$ Vercauteren et al demonstrated that the combination of sufentanil with tramadol reduced the dose requirements of both components by approximately $20 \%{ }^{27}$ In comparison with another study conducted at our institution, the consumption of tramadol in the present study was reduced by about half, even though there was no significant differences in sufentanil consumption between the two studies. ${ }^{20}$ One clinical trial found that tramadol is 10 times less potent than morphine, ${ }^{28}$ and morphine is $400-1,000$ times less potent than sufentanil. ${ }^{29}$ Thus, $1 \mathrm{mg}$ of sufentanil could be considered equipotent to 4,000-10,000 mg of tramadol. If we consider $10 \mathrm{mg}$ tramadol as equivalent to $1.5 \mu \mathrm{g}$ sufentanil, the consumption of sufentanil during the first 24 hours was about $121 \mu \mathrm{g}$ in the present study. The incidence of side effects showed no increase in comparison to the previous study conducted at our institution. In this way, the combination of sufentanil and tramadol in our study was safe.

The median time to initiation of lactation was 55-56 hours postoperatively. ${ }^{12}$ The PCA was terminated 24 hours after surgery in the present study. Based on the pharmacokinetics of sufentanil (the mean elimination half-life was 784 minutes when an intravenous bolus injection of $250-1,500 \mu \mathrm{g}$ sufentanil was administered) and tramadol ( $t_{1 / 2} \approx 6$ hours $),{ }^{30}$ both drugs may have been partially eliminated during lactation. ${ }^{31}$ The amount of colostrum available within the first few days after delivery is relatively small; thus, the amount of the drug transferred in the milk is also relatively small. ${ }^{32}$

In consideration of any perceived risk of infection, the mother's inconvenience, and replacement, dislocation, or blockage of the catheter, PCEA is not a favorable option for cesarean delivery. Therefore, at our institution, epidural catheters are routinely removed at the end of the CS procedure.

This study has several limitations. First, it is a retrospective study; therefore, blinding and randomization were practically impossible. These aspects limited the rigor of the study. Second, operations within the three groups were not performed within the same year. Furthermore, the intensity of postoperative pain might have been influenced by other factors such as the development of CS technology. Third, sufentanil and tramadol are lipophilic and can be potentially stored in fatty breast tissue and slowly released into breast milk. The concentrations of sufentanil and tramadol in the plasma and breast milk could not have been accurately measured. Generally, a retrospective study design such as that employed in the present study always has a substantial risk of bias. It did not facilitate the formulation of conclusive comments on the relevant cause and effect relationships. Therefore, we should be cautious in interpreting the data. A further prospective randomized trial is required to verify these results.

\section{Conclusion}

The data in the present study indicate that the VAS scores for postoperative pain decreased as the concentrations of sufentanil and tramadol were moderately increased within 24 hours after surgery. Moreover, the total consumption of sufentanil was reduced, without any increase in the incidence of side effects.

By adjusting the concentrations of sufentanil-tramadol combinations, the postoperative analgesic effect could be improved.

\section{Acknowledgment}

The authors wish to acknowledge our colleague Dr Shanna Guo for her assistance with the study.

\section{Disclosure}

The authors report no conflicts of interest in this work.

\section{References}

1. Gerbershagen HJ, Aduckathil S, van Wijck AJ, Peelen LM, Kalkman CJ, Meissner W. Pain intensity on the first day after surgery: a prospective cohort study comparing 179 surgical procedures. Anesthesiology. 2013;118(4):934-944.

2. Nikolajsen L, Sørensen HC, Jensen TS, Kehlet H. Chronic pain following Caesarean section. Acta Anaesthesiol Scand. 2004;48(1): 111-116.

3. Sutton CD, Carvalho B. Optimal pain management after cesarean delivery. Anesthesiol Clin. 2017;35(1):107-124.

4. Kuczkowski KM. Postoperative pain control in the parturient: new challenges in the new millennium. J Matern Fetal Neonatal Med. 2011;24(2):301-304.

5. Moustafa AA, Baaror AS, Abdelazim IA. Comparative study between nalbuphine and ondansetron in prevention of intrathecal morphineinduced pruritus in women undergoing cesarean section. Anesth Essays Res. 2016;10(2):238-244.

6. Sultan P, Halpern SH, Pushpanathan E, Patel S, Carvalho B. The effect of intrathecal morphine dose on outcomes after elective cesarean delivery: a meta-analysis. Anesth Analg. 2016;123(1):154-164.

7. Vercauteren M, Vereecken K, La Malfa M, Coppejans H, Adriaensen H. Cost-effectiveness of analgesia after caesarean section. A comparison of intrathecal morphine and epidural PCA. Acta Anaesthesiol Scand. 2002;46(1):85-89.

8. Miaskowski C. Patient-controlled modalities for acute postoperative pain management. J Perianesth Nurs. 2005;20(4):255-267. 
9. Modak SD, Kane DG. Conscious sedation for balloon mitral valvotomy: a comparison of fentanyl versus sufentanil. Ann Card Anaesth. 2017;20(2):163-168.

10. Andziak M, Beta J, Barwijuk M, Issat T, Jakimiuk AJ. Efficacy and tolerability of intravenous morphine patient-controlled analgesia (PCA) in women undergoing cesarean delivery. Ginekol Pol. 2015;86(6):453-456.

11. Palmer PP, Miller RD. Current and developing methods of patientcontrolled analgesia. Anesthesiol Clin. 2010;28(4):587-599.

12. Nie Y, Liu Y, Luo Q, Huang S. Effect of dexmedetomidine combined with sufentanil for post-caesarean section intravenous analgesia: a randomised, placebo-controlled study. Eur J Anaesthesiol. 2014;31(4):197-203.

13. Zhang Y, Duan G, Guo S, et al. To predict sufentanil requirement for postoperative pain control using a real-time method: a prospective observational cohort study. Medicine (Baltimore). 2016;95(25):e3915.

14. Gadsden J, Hart S, Santos AC. Post-cesarean delivery analgesia. Anesth Analg. 2005;101(5 Suppl):S62-S69.

15. Wilder-Smith CH, Hill L, Wilkins J, Denny L. Effects of morphine and tramadol on somatic and visceral sensory function and gastrointestinal motility after abdominal surgery. Anesthesiology. 1999;91(3):639-647.

16. Raffa RB, Friderichs E, Reimann W, Shank RP, Codd EE, Vaught JL. Opioid and nonopioid components independently contribute to the mechanism of action of tramadol, an 'atypical' opioid analgesic. $J$ Pharmacol Exp Ther. 1992;260(1):275-285.

17. Lopopolo M, Affaitati G, Fabrizio A, et al. Effects of tramadol on viscero-visceral hyperalgesia in a rat model of endometriosis plus ureteral calculosis. Fundam Clin Pharmacol. 2014;28(3):331-341.

18. Ribezzi M, Di Venosa N, Nicoletti E, Lauta E, Giuliani R. The association of tramadol and morphine in the treatment of acute postoperative pain. Minerva Anestesiol. 2011;77(2): 239; author reply 240-241.

19. Guo S, Duan G, Wang J, Chi X, Zhang L, Zhang X. [Comparison of sufentanil-tramadol PCIA between laparoscopic cholecystectomy and gynecological laparoscopy]. Zhonghua Wai Ke Za Zhi. 2015;53(2): 150-154 [in Chinese].

20. Chi X, Li M, Mei W, Liao M. Comparison of patient-controlled intravenous analgesia with sufentanil versus tramadol in post-cesarean section pain management and lactation after general anesthesia - a prospective, randomized, double-blind, controlled study. J Pain Res. 2017;10:1521-1527.
21. Wang C, Li L, Shen B, et al. A multicenter randomized double-blind prospective study of the postoperative patient controlled intravenous analgesia effects of dezocine in elderly patients. Int J Clin Exp Med. 2014;7(3):530-539.

22. Vadivelu N, Chang D, Helander EM, et al. Ketorolac, oxymorphone, tapentadol, and tramadol: a comprehensive review. Anesthesiol Clin. 2017;35(2):e1-e20.

23. Mitra S, Khandelwal P, Sehgal A. Diclofenac-tramadol vs. diclofenacacetaminophen combinations for pain relief after caesarean section. Acta Anaesthesiol Scand. 2012;56(6):706-711.

24. Demirel I, Ozer AB, Atilgan R, et al. Comparison of patient-controlled analgesia versus continuous infusion of tramadol in post-cesarean section pain management. J Obstet Gynaecol Res. 2014;40(2): 392-398.

25. Owusu Obeng A, Hamadeh I, Smith M. Review of opioid pharmacogenetics and considerations for pain management. Pharmacotherapy. 2017;37(9):1105-1121.

26. Santos Garcia JB, Lech O, Campos Kraychete D, et al. The role of tramadol in pain management in Latin America: a report by the Change Pain Latin America Advisory Panel. Curr Med Res Opin. 2017;33(9):1615-1621.

27. Vercauteren MP, Mertens E, Schols G, Mol IV, Adriaensen HA. Patientcontrolled extradural analgesia after caesarean section: a comparison between tramadol, sufentanil and a mixture of both. Eur J Pain. 1999;3(3):205-210.

28. Casali R, Lepri A, Cantini Q, Landi S, Novelli GP. [Comparative study of the effects of morphine and tramadol in the treatment of postoperative pain]. Minerva Anestesiol. 2000;66(3):147-152 [in Italian].

29. Cafiero T, Di Minno RM, Sivolella G, Di Iorio C. Immediate postoperative pain management in patients undergoing major abdominal surgery after remifentanil-based anesthesia: sufentanil vs tramadol. Minerva Anestesiol. 2004;70(9):661-669.

30. Grond S, Sablotzki A. Clinical pharmacology of tramadol. Clin Pharmacokinet. 2004;43(13):879-923.

31. Hendrickson RG, McKeown NJ. Is maternal opioid use hazardous to breast-fed infants? Clin Toxicol (Phila). 2012;50(1):1-14.

32. Carvalho B, Butwick AJ. Postcesarean delivery analgesia. Best Pract Res Clin Anaesthesiol. 2017;31(1):69-79.

\section{Journal of Pain Research}

\section{Publish your work in this journal}

The Journal of Pain Research is an international, peer reviewed, open access, online journal that welcomes laboratory and clinical findings in the fields of pain research and the prevention and management of pain. Original research, reviews, symposium reports, hypothesis formation and commentaries are all considered for publication.

\section{Dovepress}

The manuscript management system is completely online and includes a very quick and fair peer-review system, which is all easy to use. Visit http://www.dovepress.com/testimonials.php to read real quotes from published authors. 